EDITORIAL

\title{
Global Perspectives on Music Therapy
}

\author{
Katrina McFerran ${ }^{1}$ * \\ 1 University of Melbourne, Australia \\ *k.mcferran@unimelb.edu.au
}

Published: 1 March 2019

We welcome contributions from around the globe and our editorial team works tirelessly with authors so that their ideas can be shared with the community of music therapists and others interested in music, health, and social change. We are proud of this issue of Voices, since it contains contributions from seven different countries. The authors' accents can be heard and are celebrated in each article, both in the way they phrase their ideas and the theoretical foundations they draw upon to describe their work and research. This geographical diversity goes some way towards achieving the mission of Voices, and concretely demonstrates our values of inclusion and socio-cultural awareness. It is also a part explanation of why a number of articles in this edition have been in production for some time, despite our aspiration of moving swiftly through review and into open access, online production. Diversity does not always result in efficiency, but we believe the extra time spent by everyone involved compensates for that, and hope you agree.

In addition to representing different geographic regions, the articles in the March issue also represent different traditions of research and evaluation. Mitchell (from Canada) draws on ethnomusicology as one of the frames of reference for her investigation, which she positions as a participatory project. Knapik-Szweda (from Poland) describes music therapy as an interdisciplinary branch of science and uses qualitative data collection strategies including interviews and observations to evaluate the use of an assessment tool. Chadder (from the UK) uses a mixed methods approach, which involved both online survey responses and interviews, which she follows up with professional development programs to address gaps in knowledge. Hannibal and colleagues (from Denmark) systematically pilot and attempt to manualise an intervention which can subsequently be used in a randomized, controlled trial. Hense (from Australia) describes her work as a practice-based study utilizing mixed data which is analysed for themes and results in recommendations for youth practice. In Reflections on Practice, Kwoun (from the USA) evaluates the development of music therapy students in a community music therapy project. We also include a book review in this edition, where Tahara (from Japan) provides both English and Japanese reflections on A History of the Music Therapy Profession and concludes that Byers does offer perspectives that are relevant across cultures.

The intersection of different perspectives continues throughout the edition, from contexts where music therapy is practiced to the people who are participating in the various music programs and projects. At one end of the spectrum, Hense's work is located in a community context where she intends to support youth as they transition 
out of institutional mental health contexts. At the other, Hannibal's work describes clients who have had more than 40 sessions of analytically oriented music psychotherapy and mentalization-based treatment as part of inpatient mental health treatment. In between is Mitchell's exploration of a performance event held by an adolescent mental health treatment facility with an ethos that levels power dynamics between young people and workers within the institution. In the disability field, Knapik-Szweda focuses on two children with multiple disabilities to understand whether individual music therapy improves social and communicative functioning. In contrast, Kwoun positions the people with disabilities as teachers of her music therapy students who participate in a program run with a local symphony orchestra. Hadder draws on her background as a community musician to investigate the perceptions of health care staff on the value of what she calls music interventions, and is surprised to learn that most do not know anything about how music might be helpful, and some do not wish to learn more.

This edition is an exciting representation of diversity in our global practices, but it is still dominated by the faces of white, western women, who are also the majority of the international profession. So, we have come some way towards inclusive practices, but there is still some distance to travel before we can truly be considered diverse. There are also many intersections among authors. The commitment to understanding how music works, when it is helpful, and who might benefit from it if they are granted access is common across the contributions. In addition, the effort behind each one of these articles, first in the doing of the research or evaluation, and subsequently in writing it up and participating in a rigorous review process to ready it for publication, is enormous. We are proud to share this collection and look forward to hearing more perspectives from contributors in the future so that we may continue this tradition of providing an egalitarian and interdisciplinary forum so that multiple voices can be heard. 